\section{REVISTA}

Actualidades Investigativas en Educación http://revista.inie.ucr.ac.cr/

ISSN 1409-4703

\title{
PRÁCTICAS LECTORAS DE ESTUDIANTES UNIVERSITARIOS CON FINES DE ESCRITURA ACADÉMICA
}

READING PRACTICES FOR ACADEMIC WRITING PURPOSES ON COLLEGE STUDENTS

\author{
Volumen 16, Número 1 \\ Enero - Abril \\ pp. 1-19
}

Este número se publicó el $1^{\circ}$ de enero de 2016

DOI: http://dx.doi.org/10.15517/aie.v16i1.21971
Yolanda González de la Torre José Jiménez Mora Jorge Ignacio Rosas

Revista indizada en REDALYC, SCIELO

Revista distribuida en las bases de datos:

LATINDEX, DOAJ, E-REVIST@S, IRESIE, CLASE, DIALNET, SHERPA/ROMEO, QUALIS,

Revista registrada en los directorios:

ULRICH'S, REDIE, RINACE, OEI, MAESTROTECA, PREAL, CLACSO 


\title{
PRÁCTICAS LECTORAS DE ESTUDIANTES UNIVERSITARIOS CON FINES DE ESCRITURA ACADÉMICA \\ READING PRACTICES FOR ACADEMIC WRITING PURPOSES ON COLLEGE STUDENTS
}

\author{
Yolanda González de la Torre ${ }^{1}$ \\ José Jiménez Mora² \\ Jorge Ignacio Rosas ${ }^{3}$
}

\begin{abstract}
Resumen: Dado que en el nivel de la Educación Superior se espera un desempeño competente de los estudiantes en tareas de lectura y escritura, la experiencia en diversas habilidades lingüísticas permite reconocer deficiencias importantes en los estudiantes, las cuales han dado lugar a distintas propuestas pedagógicas de la lectura y la escritura universitaria. Con el fin de aportar datos acerca de la problemática que experimentan los estudiantes en la práctica lectora cuando se orienta a la escritura de un texto académico, en este artículo se discuten algunos resultados del desempeño de estudiantes de licenciatura en un área de Ciencias Sociales de una universidad pública de la ciudad de Guadalajara, Jalisco, México. La metodología empleada es de orden mixto. En un primer momento, mediante un análisis de tarea se examinan textos elaborados por los estudiantes en los cuales reportan un breve estado de la cuestión sobre una temática, y posteriormente se analizan datos de una entrevista relacionados con la experiencia lectora de los estudiantes en este tipo de tarea académica. Los resultados muestran cómo los estudiantes realizan algunos procesos como el reconocimiento de ideas clave, la asociación temática o la síntesis, pero también que experimentan dificultades como la articulación de las ideas o la apropiación de argumentos. Una de las conclusiones principales del trabajo es que estos procesos y dificultades se vinculan fuertemente con el tipo de demandas de lectura que tienen los estudiantes de acuerdo con su contexto escolar.
\end{abstract}

Palabras clave: LECTURA ACADÉMICA, ESCRITURA ACADÉMICA, ESTUDIANTES UNIVERSITARIOS, MÉXICO.

\begin{abstract}
The higher education reading and writing tasks are expected to be performed by students in a competent way. However, experience in various fields allowed major deficiencies in students, which have led to various proposals for teaching reading and writing university. In order to provide data about the problems experienced by students in reading practice, when oriented to writing an academic text, this article examines some undergraduate students' performance results are discussed in social sciences in a public university in the city of Guadalajara, Jalisco, Mexico. The methodology used was mixed order. Firstly, it was analyzed the text produced by the students in which they reported a brief Literature Review on a subject. Therefore, an interview data was analyzed using students' reading experience in academic tasks. The results show how students manage to make some processes such as the recognition of key ideas, themes association or synthesis, but also how they experience difficulties such as joint ownership of ideas or arguments. The study showed, as one of the main conclusions, that these processes and difficulties are deeply related to reading demands according to students' scholar context.
\end{abstract}

Keywords: ACADEMIC READING, ACADEMIC WRITING, COLLEGE STUDENTS, MEXICO.

\footnotetext{
1 Profesora-Investigadora del Departamento de Estudios en Educación de la Universidad de Guadalajara, México. Doctora en Educación. Dirección electrónica:ygtorre@hotmail.com

2 Profesor del Centro de Bachillerato Tecnológico, Industrial y de Servicios No. 10 de la Secretaría de Educación Pública, México Maestro en Investigación en Ciencias de la Educación. Dirección electrónica: pepe dw@hotmail.com

${ }^{3}$ Coordinador de Investigación del Centro Universitario del Norte, Universidad de Guadalajara, Colotlán, Jalisco, México. Maestro en Investigación en Ciencias de la Educación. Dirección electrónica: transmodernidad@hotmail.com
}

Artículo recibido: 26 de mayo, 2015

Enviado a corrección: 12 de agosto, 2015

Aprobado: 9 de noviembre, 2015 


\section{Introducción}

La lectura y la escritura son procesos fundamentales empleados para intercambiar ideas y conocimientos. El desarrollo de tales procesos constituye un soporte para la adquisición de aprendizajes y el desarrollo de la ciudadanía que requiere la sociedad. Sin embargo, en diferentes escenarios o instituciones (escuela, trabajo, bibliotecas) tanto la lectura como la escritura se definen, utilizan y evalúan de manera particular, como han señalado en distintos momentos Caldera y Bermúdez (2007) y Cook-Gumperz (1988), y entre otros. Asimismo, fenómenos como la proliferación de tecnologías de la información y comunicación están provocando una dinámica plural de textos y modos de leer, de tal modo que en la escuela y fuera de ella, muchos aprendizajes se obtienen y canalizan a través del texto escrito en diversos formatos, desde impresos hasta telemáticos (Luke, 2005; Solé y Castells, 2004).

Sin embargo, la disponibilidad de nuevos medios para acceder a la lectura y la escritura no garantiza por sí sola que las personas se vuelvan más competentes para estas tareas, en particular, en los contextos escolares. En este sentido, trabajar como docente en un curso con estudiantes de pregrado constituye toda una experiencia, pues es posible encontrar fenómenos como el choque de expectativas entre quienes desempeñan roles docentes y el estudiantado. Como consecuencia, hay quejas en el profesorado acerca de la falta de ciertas habilidades de lectura que suponen el estudiantado tendría que haber desarrollado previamente, como identificar conceptos y argumentos, expresarse de manera clara en forma oral y escrita, y en general, aquellas que tienen que ver con la comprensión de textos (Carlino, 2004).

Poner atención en las deficiencias de lectura de las estudiantes y de los estudiantes percibidas por el profesorado, ha hecho que en nuestra experiencia en ciertos cursos repetidamente se deba realizar trabajo de carácter remedial, entre otras cosas, encaminado a que el estudiantado adquiera capacidad para recuperar y comprender la información que lee y el uso que de ésta debe hacer para la construcción de textos académicos.

\section{El enfoque de este estudio}

En este trabajo un punto de partida es considerar a la lectura y la escritura no solo como prácticas cognitivas, sino también sociales. Tal perspectiva reconoce el contexto como un elemento importante en la adquisición y desarrollo de tales actividades. En este sentido, los entornos o contextos no se encuentran vacíos de significados sociales y culturales, sino 
que otorgan ciertos rasgos a la forma de concebir los usos y objetivos de la lectura y la escritura, que las hace adquirir ciertos matices en un espectro de cualidades diversas (Barton, 1994; Cook-Gumperz, 1988; Diehl y Mikulecky, 1988; González, 2011; Kalman, 2004; Rockwell, 1988; Sticht, 1978; Street, 2004; Szwed, 1988; Verhoeven, 1994). De igual forma, estudios como los citados reconocen particularidades en las personas que leen y escriben que comprenden la participación de procesos cognitivos individuales en el desarrollo de la lectura y la escritura, así como habilidades y estrategias que en cada sujeto se manifiestan de acuerdo con sus formas de interpretar la exigencia del contexto en que realiza tareas de lectura y escritura (Castelló, 2002; Hamilton, 2000; Zavala, 2011). En suma, como sostiene Carlino (2003a), las formas de buscar, adquirir, elaborar y comunicar conocimiento, es decir, los modos de leer y escribir no son iguales en todos los ámbitos. No obstante, es innegable que en el nivel de estudios universitario la lectura y la escritura son herramientas para la construcción de conocimiento y para dar cuenta de su adquisición y desarrollo.

Dentro de un escenario escolar específico como el que forma parte de este estudio, se puede cuestionar en qué medida el profesorado incorpora en la práctica con sus estudiantes formas de leer y escribir necesarias no solo para adquirir, sino para construir conocimiento. Carlino (2003b) ha encontrado que no siempre el profesorado define las tareas solicitadas de manera clara, específicamente cuando se trata de inducir al estudiantado a demandas de lectura como las de encontrar en los textos científicos y académicos las distintas posturas o argumentos que sostienen o sus relaciones con otros textos, lo cual implica una búsqueda que exige operar sobre el texto mediante acciones específicas que caracterizan a una determinada comunidad lectora.

De este modo, las prácticas lectoras del estudiantado tienen que ver con los fines, usos y costumbres que adopta como resultado de ciertas prioridades de trabajo, en este caso, en un contexto como el de un aula universitaria. Por ejemplo, si durante la clase hay que repetir ideas de un texto asignado por la profesora o profesor como evidencia de que se cumplió con la tarea asignada, esa acción puede ser suficiente para el estudiantado, por encima de la reflexión y el análisis sobre el texto.

En relación con lo anterior, estudios como el de Zavala (2011) han encontrado que las y los estudiantes no incorporan de manera homogénea las formas y usos de los textos académicos, en lo cual influyen sus experiencias en grados previos y la comprensión que tienen al respecto. De modo similar, Muñoz y Maldonado (2013) señalan que la mayor 
dificultad que tienen las estudiantes y los estudiantes es la identificación de conceptos que deben estar en los escritos, proceso que se ve influido por las consignas que establece el profesorado respecto al tipo de texto a producir y sus características.

Como se aprecia en los elementos presentados anteriormente, problemáticas que se asocian a las prácticas lectoras de estudiantes universitarios no solo tienen que ver con cuestiones cognitivas, sino con otras de tipo sociocultural, como las condiciones impuestas por las profesoras y los profesores en torno a las acciones a realizar por el estudiantado con los textos. En este sentido, el desempeño lector que es posible notar en estudiantes de este nivel educativo ante demandas de escritura académica puede estar afectado por algunas prácticas arraigadas en el profesorado. Por ello es necesario acercarse a un objeto de estudio como ese desde ambas perspectivas, cognitiva y sociocultural. De allí que esta investigación se haya orientado por las siguientes preguntas:

¿Qué características de las prácticas lectoras son identificables a partir de la revisión de los escritos producidos por estudiantes de licenciatura en los cuales reportan un estado de la cuestión?

¿Qué rutinas y normas de trabajo en el aula universitaria pueden influir sobre la elaboración de este tipo de textos por parte de las estudiantes y los estudiantes?

¿Cuáles de las insuficiencias percibidas por el profesorado o de las dificultades presentes en el estudiantado pueden ser atribuidas a aspectos propios del contexto de desempeño universitario, en el caso en estudio?

\section{Metodología. La escritura como evidencia de prácticas lectoras situadas}

Para construir explicaciones acerca de los aspectos abordados en las preguntas anteriores, se realizó un estudio mixto, que contempla por un lado analizar los textos que producen los estudiantes posteriores a tareas de lectura, y por otro, indagar sus percepciones en torno a factores del contexto que intervienen en sus prácticas lectoras. Los instrumentos, que serán descritos más adelante, permiten dar cuenta de algunos rasgos particulares de esta actividad en los contextos donde se desenvuelve el estudiantado.

\subsection{Participantes, contexto y acopio de datos}

Fueron incluidos cinco estudiantes, dos mujeres y tres hombres, quienes formaban un grupo de la licenciatura en Sociología inscrito en un curso relacionado con temas de la investigación educativa, en una universidad pública del occidente de México. Se trata de un 
muestreo por conveniencia, dado que la integración del grupo de sujetos se dio porque eligieron un curso en el penúltimo semestre de su formación escolarizada referente a temas de investigación educativa. Las sesiones de clase, una por semana, tuvieron una duración de cuatro horas cada una, y en ellas se trabajó en la discusión de una lectura propuesta por la profesora acerca de temas relacionados con el contenido del curso.

Como parte de las actividades del curso, el estudiantado realizó cada semana la lectura de diversos artículos en los cuales se presentaran reportes de investigación, con el fin de familiarizarse con este tipo de textos y al mismo tiempo apropiarse de información útil para clarificar sus temas de tesis. Parte del desarrollo de la clase consistió entonces en que cada estudiante expusiera de manera oral una síntesis de algún artículo revisado. Como producto final del curso tenían que construir un breve estado de la cuestión basado en la lectura de tales artículos. Para esta tarea, la profesora previamente las había explicado en detalle las características de un texto académico de este tipo y las formas de búsqueda y recuperación de información clave de cada documento.

Los textos elaborados por las y los discentes, como estado de la cuestión, sirven en el presente estudio para encontrar datos en torno a su actividad lectora, partiendo del supuesto de que esta se lleva a cabo con fines específicos (Barton y Hamilton, 1998; Barton y Hamilton, 2000), y en este sentido el producto que escriben refleja ciertos énfasis de la práctica lectora, producto de los usos y costumbres que dan a los textos en su contexto escolar cotidiano.

Las estudiantes y los estudiantes de este contexto se formaban como profesionales en un área determinada de conocimiento, las ciencias sociales. Esto se traduce en la presencia de ciertas demandas de actividad lectora, las cuales tienen relación con el uso de un discurso peculiar, predominantemente expositivo, en el que la información hace referencia a hechos e ideas, emplea conceptos complejos y vocabulario especializado, al grado de contener párrafos completos para conceptualizar o definir términos. Otras de las demandas que supone la lectura en este tipo de contexto son establecer la secuencia cronológica de eventos importantes, comparar ideas y predecir resultados (Escalante y Caldera, 2006).

Parte de la exploración de las acciones que el estudiantado realiza para cumplir con la consigna que se señaló, construir un estado de la cuestión, se sostiene en este trabajo en el método llamado análisis de tarea. Este método, de acuerdo con Rodrigo (1982), permite desglosar las acciones en componentes más simples, entre los que se encuentran: 
- Las destrezas generales del sujeto para la resolución de la tarea.

- Las herramientas conceptuales que posee.

- La utilización de dichas herramientas en función de las características de la tarea.

- La capacidad de planificación del sujeto en el proceso de resolución.

Este método funciona como un modo de observación que permite dar cuenta de características o rasgos relacionados directamente con las acciones que el sujeto lleva a cabo para la resolución de determinadas tareas. Como consecuencia, define normas y criterios de actuación que se esperan de parte de las y los sujetos en condiciones ideales, o rutas de desempeño seguidas por "expertos" (Dueñas y Jiménez, 2006).

En el caso de la construcción de un estado de la cuestión, una de estas rutas implica, por un lado, seleccionar información pertinente de diversos artículos de investigación leídos, para dar cuenta de manera escrita de las aportaciones en torno a una temática u objeto de estudio. Por otro lado, tal información se tiene que articular por escrito a manera de una discusión en la que se abordan aspectos teóricos y empíricos, explicando las tendencias predominantes sobre la temática e incluyendo preguntas que se derivan de los elementos no resueltos en tales artículos. Si bien esta segunda parte de la tarea involucra acciones de escritura, las acciones de lectura implicadas en un desempeño "ideal" del estudiantado pueden alcanzar, desde la perspectiva de este trabajo, tres niveles progresivos, a saber:

a. Síntesis. Se trata de que la estudiante o el estudiante resuman la información que leyeron, aun cuando la reporten de manera literal. Un ejemplo de evidencia de que arriban a este nivel de lectura es, como se verá más adelante, el recorrido que el estudiantado hace en los textos que construye por partes clave de los artículos de investigación que revisa, como sus objetos de estudio, sus instrumentos y algunos resultados destacados.

b. Apropiación coherente de significado. Refiere a la posibilidad de que la estudiante o el estudiante muestren una comprensión precisa de las ideas que leen. Un ejemplo de evidencia de esta acción lo constituye el modo de hacer referencia a los autores. Cuando algún(a) estudiante dice en su texto construido, por ejemplo, "como lo menciona", en lugar de "sostiene", o "afirma", es posible inferir en el análisis de su texto que no entiende bien la naturaleza del documento que reporta, porque está tomando una idea de carácter argumentativo como una de otro tipo, más de carácter descriptivo o expositivo. 
c. Identificación de asociación temática. Se refiere al hecho de que la estudiante o el estudiante puedan apreciar ideas de distintos textos que estén relacionadas. Un ejemplo de evidencia en este sentido es que en un mismo párrafo citen a varios autores en razón de que hablan de un tema común.

En un segundo momento, para dar cuenta de cómo ciertas prácticas lectoras se pueden asociar con determinadas rutinas de trabajo con los textos en el aula, se realizó una entrevista con el estudiantado en la cual abordaron aspectos de su experiencia personal en la lectura de los artículos de investigación y la incorporación de las ideas en el texto escrito referido al estado de la cuestión. Las entrevistas se realizaron de manera individual, se audiograbaron y posteriormente fueron transcritas bajo un acuerdo de confidencialidad para el uso de la información obtenida y los trabajos elaborados, respetando el anonimato de los participantes.

Los ejes que guiaron la entrevista fueron dos:

- La práctica lectora individual. Aquí se les solicitó describir la forma en la que leyeron los textos y seleccionaron la información. En este eje se incluyen desde preguntas generales del tipo “¿Cómo lees?” “¿Tienes algún plan de lectura antes de iniciar la tarea?" Hasta otras muy puntuales, tales como “¿De qué manera identificas la información que te resulta relevante?” “¿Cómo distingues en cada texto la cantidad de información que puedes incorporar en tu trabajo?"

- Organización de la tarea a desarrollar. Este eje comprende preguntas para explorar acciones de carácter estratégico empleadas por el estudiantado para el desarrollo y concreción del estado de la cuestión, como la identificación de argumentos, posturas y hallazgos en los reportes leídos por las estudiantes y los estudiantes y su puesta en el documento, acciones que para esa tarea realizan los expertos (Salgado, 2007).

\section{Resultados. Los reflejos en los textos sobre la práctica lectora}

En el contexto del cual se obtienen los datos empíricos en este trabajo la profesora pidió durante el curso al estudiantado dirigir su actividad lectora a extraer y procesar información de artículos de investigación con tres propósitos: intervenir en forma oral en clase, producir síntesis de lectura de cada texto, y como tarea integradora, según se ha señalado, hacer un breve estado de la cuestión. Los resultados que se exponen enseguida 
se relacionan con el análisis de los textos escritos por los estudiantes como parte de esta última tarea.

De acuerdo con los datos del análisis de tarea, es posible que las estudiantes y los estudiantes consigan arribar a los dos primeros niveles de desempeño lector requeridos en la construcción del estado de la cuestión (síntesis y apropiación coherente de significado), como se observa en los fragmentos siguientes ${ }^{4}$ :

\section{Estudiante 1}

El arte de formar y la artesanía del saber la autora es María Esther Aguirre Lora,

El siguiente artículo es el resumen de un libro, en el cual la autora hace la descripción acerca de las artesanías de Chiapas de corzo, donde hace una descripción de cómo se adentra en los talleres artesanales y como es que observa a los miembros de estos talleres aprendiendo el uno del otro y compartiendo secretos de este campo, la metodología que emplea son las entrevistas y la observación, .....nos habla también acerca de que aun que esta sea una educación informal la que emplean los artesanos, tiene un gran significado que deberíamos de aprender...

\section{La formación de redes sociales en el estudio de actores y familiar. Perspectiva} de estudio en historia y Antropología. Carmen Imelda González Gómez Y Manuel Basaldúa Hernández. Redes: revista hispana para el análisis de redes sociales $N^{\circ} 12$ 2007.

El propósito de este trabajo es articular dos disciplinas sociales para mostrar que las redes sociales se convierten en un modelo dinámico en la observación y análisis de ciertos atributos de los individuos como actores singulares y en grupo, la recolección de datos fue mediante entrevistas a profundidad. Los autores de este artículo nos presenta como se dan las redes en una pequeña empresa describe como es la jerarquía dentro de esta y cuáles son los vínculos entre los miembros del grupo, y cuáles son los valores, intereses, rasgos culturales, producción y ganancias entre otros. Dice también que las primeras generaciones qué comenzaron a laborar en esta pequeña empresa fueron transmitiéndose de generación en generación. ${ }^{5}$

\footnotetext{
4 Los fragmentos anteriores se dejaron en su forma original a propósito, en parte para mostrar los modos naturales en que escriben las estudiantes y los estudiantes, pero también para apreciar las formas en que realizan la extracción de determinada información encaminada a la construcción de productos escritos.

${ }^{5}$ El subrayado que se incluye en los textos de las y los estudiantes es de los autores de este estudio, con la intención de señalar aspectos puntuales de la actividad lectora reflejados en la construcción del texto, como la copia de información textual y algunas expresiones redactadas erróneamente.
} 
En el primero de los fragmentos anteriores se distingue una rutina en la cual una estudiante cita el tipo de trabajo que revisa, así como la metodología que es empleada en ese trabajo y algunas conclusiones, por ello se considera que el primer nivel de desempeño, la síntesis de información, se cumple. Por otro lado, una evidencia de que la estudiante alcanza el segundo nivel, apropiación coherente de significado, es el hecho de que parafrasea la información que leyó. No obstante, no existe evidencia de que la estudiante consigue llegar al tercer nivel, denominado asociación temática, dado que presenta los textos por separado, aún cuando le fue indicado que intentara hilar un solo escrito a partir de una temática común de discusión. Por otra parte, en los anteriores fragmentos es posible notar una especie de extensión del discurso oral, presente en ciertas deficiencias (resaltadas en cursiva), tales como expresiones mal escritas de alguna palabra y redundancias. Esa extensión del discurso oral es más notable aún en textos como el siguiente:

\section{Estudiante 2}

Otro artículo encontrado en la red sobre problemas y perspectivas de futuro en torno a internet y las tecnologías de la información y la comunicación en las administraciones públicas del siglo XXI del Instituto Vasco de Administración Pública, nos comparte sobre las nuevas tecnologías de información referente la vialidad de difusión en internet, también menciona que a finales del siglo XX diversos autores hacen reflexiones y estudios, en donde consideran que una nueva etapa en la Historia de la Humanidad ha comenzado: la Era Informacional (Castells, 1996); que vivimos en un Mundo Digital (Negroponte, 1995) y en una Sociedad en Red (Castells, 1996) y de la Información y el Conocimiento ubicada en el Tercer Entorno (Echeverría, 2999):

El fragmento anterior es una evidencia del tipo de texto que refleja que se alcanza el primer nivel de lectura, que permite hacer la síntesis del contenido del artículo que el estudiante revisó. En este caso, hay mucha menos paráfrasis y más copia del texto, desde el título, que es indicado como una temática (primer subrayado), hasta afirmaciones completas, incluyendo citas hechas en el documento original que fue leído por el estudiante. Sin embargo, al incluir prácticamente solo información literal, no ofrece evidencia de que se haya apropiado de su significado.

Como se ve en parte de los fragmentos anteriores, en ocasiones el estudiantado está acostumbrado a transcribir grandes cantidades de información. Eso corrobora lo señalado 
por Carlino (2007) respecto a la transición de la lectura a la escritura, sobre la situación de que esta última tarea es concebida tanto por el estudiantado como por el profesorado como una cuestión de norma, de producir o reproducir texto, pero no como una manera de estructurar ideas en un género discursivo específico. Respecto a lo anterior, en el conjunto de datos fue poco el texto del estudiantado en que se consiguió detectar el tercer nivel, la asociación temática. Uno de los fragmentos donde se observa este desempeño es el siguiente:

\section{Estudiante 4}

Tanto los trabajos de Petrosino y el de Valenti y López son trabajos totalmente diferentes uno lo marca como la evolución de la educación técnica y los otros ya lo manejan como una educación diferente un educación que innova e injiere directamente en la sociedad pero los dos trabajos casi no tocan muy a fondo la formación humanística y social que para mi el tecnólogo debe tener.

Comparar dos trabajos - aún cuando la estudiante comete el error de no referenciar el año - en cuanto a sus perspectivas, es uno de los rasgos de lo que en este trabajo se denomina nivel de asociación temática. En otros casos, una evidencia del logro de este nivel es que la estudiante o el estudiante, junto con la relación de ideas, se refieran a los trabajos en su conjunto, a la par que hacen una breve descripción de su construcción y un juicio sobre la aportación al conocimiento, como se muestra enseguida:

\section{Estudiante 3}

Los autores que se enfocaron en el aprendizaje fueron, Vargas (2003) a quien le interesaba aclarar los conocimientos que posee y deben poseerlos jóvenes sobre el desarrollo moral, valores y ética. Olivera Stella y otros (2005), le interesaba conocer los valores que influyen en la formación ciudadana en escuelas secundarias. Y el último Miraflores Gómez (2006), a quien le interesaba comprobar si el alumnado (futuros maestros y educadores) percibían o no que sus profesores, transmiten unas actitudes y unos valores en la acción docente.

Mientras que Genaro Meza Hernández (2008), se enfoco, en observar, analizar e interpretar como inciden las actitudes del docente en la enseñanza de valores, es decir, en el aprendizaje de los alumnos. Compartiendo rumbo de su investigación con Gómez 
López (2005), a quien le fue de importancia los métodos utilizados por el profesor y si estos van acorde con la Universidad.

Estos trabajos muestran la definición que los niños le han otorgado a los valores, así como cuales no han obtenido definición alguna. La redacción y comienzo de las investigaciones marcharon de distinta forma, ya que por un lado unas iniciaban con historia breve del objeto de estudio en cuestión, otras más con el planteamiento actual del objeto a investigar.

Salgado (2007) sostiene que un aspecto clave para realizar un estado de la cuestión se refiere a hacer una lectura que permita contrastar o comparar los estudios revisados para ubicar asuntos, enfoques o ámbitos relacionados con el problema de estudio. En este sentido, no todo el estudiantado consigue mostrar evidencia de un tipo de lectura en la cual alcance a realizar esos procesos correspondientes al nivel de asociación temática.

El autor citado en el párrafo anterior sostiene que, como parte final en la construcción de un estado de la cuestión, se requiere de una lectura analítica de las corrientes teóricas, modelos o categorías conceptuales, en la cual se examine la información a fin de reconstruirla alrededor de un objetivo central. En este sentido, un problema que puede inferirse a partir del análisis de los textos que no alcanzan el tercer nivel de lectura es que algunos(as) estudiantes posiblemente no advierten un hilo conductor en la temática que recuperan, por lo cual eso no se aprecia en el texto que construyen.

\section{Rutinas lectoras ante escenarios inesperados}

Desde su formación en la escuela básica, las estudiantes y los estudiantes de contextos, como el considerado en este estudio, son instruidos a leer con énfasis en recuperar ideas importantes de los textos. Eso posiblemente les conduce a que entre las estrategias de lectura incorporen aquellas que tienen que ver con la selección de información y con una comprensión de su contenido que les permita saber si se refiere a determinado tema. Cuando acceden a la formación universitaria, la práctica de la lectura y la escritura implica, además, apropiarse de la manera en que se usa la información en cada disciplina, para dar cuenta de que se han adquirido modos de participación en una comunidad académica (Cartolari y Carlino, 2011). En el caso del estudio que aquí se presenta, en la tarea escrita solicitada al estudiantado, se ve que en ocasiones este no sigue tales modos en lo referente, por ejemplo, a las normas de citado - como se aprecia enseguida: 


\section{Estudiante 5}

Se ha desprotegido a los medianos y pequeños productores y sobre todo a las agriculturas campesinas. Al integrarse al mercado agrícola internacionalizado no se puede competir con los altos subsidios que los países desarrollados brindan a sus agricultores, sobre todo a la producción de exportación.

Desde de la década d los 50's el agro mexicano registra un grave deterioro, fenómeno al que contribuyera, en aquel entonces, el impulso que se le diera al patrón de acumulación de capital, la industrialización del país, la situación de importaciones.

En el anterior fragmento del texto de una estudiante se aprecia cómo inicia con una paráfrasis de las ideas de los autores leídos y luego presenta un extracto textual, el cual indica en cursivas, pero sin citar la fuente, aunque previamente se le había explicado cuándo y cómo hacerlo. Sobre ese punto, en las entrevistas realizadas se pudo indagar cómo el estudiantado experimenta una confrontación entre sus prácticas lectoras previas y las requeridas para su desempeño en un campo disciplinar, de donde se desprende este tipo de dificultad en el tratamiento de los contenidos de los textos, como describe una estudiante:

\footnotetext{
Estudiante 1

... siendo sincera las lecturas de otras materias se me hacen muy difíciles, hay conceptos que no entiendo y se me hace muy difícil y a veces leo porque tengo que hacerlo pero no porque diga "jah, está super interesante!" [...] en sociología la mayor parte de las lecturas son teóricas, por lo que sentía que era el contenido como muy difícil...
}

Sin embargo, el estudiantado puede realizar otras acciones pertinentes para la tarea de construir un estado de la cuestión, por ejemplo, poner atención en la información y realizar correcciones personales, lo cual es indicio del aprendizaje de una lectura que se enfoca hacia la escritura, y que se caracteriza, entre otras cosas, por habilidades de planificación, monitoreo y autocorrección, tal como explica la estudiante mencionada:

\section{Estudiante 1}

...si veía que un texto me iba a servir para el trabajo así lo iba leyendo y decía "me va a servir o no me va a servir", y veía "esto si me sirve" y lo marcaba con una 
palomita...de la lectura sacar los puntos principales como palabras claves, sus objetivos, la metodología que se usó para hacer esa investigación eran como los puntos principales que teníamos que recuperar y de ahí mismo pues sacar lo que servía para nuestro tema... se me hacía más sencillo, sigo teniendo dificultades en escribir pero veía como lo hice en el reporte, cómo lo redacté y traté de corregir los errores.

Por otra parte, la comprensión del sentido de las tareas de lectura para la construcción de un estado de la cuestión, así como parte de la necesidad de realizar asociación temática entre las ideas, es expresada así por otra estudiante:

\section{Estudiante 2}

Bueno pues era leer algunos textos debía ubicar lo que decían algunos autores, ir escribiéndolo y ya tratar de compararlos con otros, no era poner lo que yo opino o estoy de acuerdo o en desacuerdo era poner lo que decían los otros para yo darme la idea de lo que tenían que hacerse el trabajo, siento que no tengo una estructura, por decir "ya terminé esto, ahora voy con lo otro", sino que conforme voy leyendo van saliendo nuevas cosas y voy adelantando y si queda un poco inconcluso voy leyendo otras cosas pero siento que no voy perdiendo el hilo.

De este corte son las explicaciones que las estudiantes y los estudiantes emiten cuando se les cuestiona cómo proceden en sus lecturas para poder resolver las tareas que el profesorado les solicita, sin dejar de lado la posibilidad de que enfrenten problemas como la falta de estrategias para articular ideas centrales de los textos que analizan, como han mostrado Peredo, Leal, Greybeck, Zarabozo y González (2007) y González (2011). Sobre este punto, uno de los estudiantes explica algunos criterios que toma en cuenta para identificar ideas centrales en los textos que lee:

Estudiante 3

I. Y entonces ¿cómo le hacías para hacer tus trabajos finales?

E. Pues del resumen que hacía de esa lectura después de leerla, en eso me basaba.

I. ¿Qué información incorporabas a tu trabajo?

E. Lo que yo consideraba que era lo más importante de la lectura.

I. ¿Y cómo decidías qué era lo más importante? 
E. Ciertas palabras clave que se adecuaban al texto, como algunos conceptos... ya cuando eran cosas como de historia yo sentía que no tenía relevancia según lo que necesitaba para el trabajo.

En lo que refiere a la planificación de su escrito, el estudiantado refiere cómo ese proceso se orienta más a la distribución del tiempo en función de las tareas que tienen que atender como parte de su actividad escolar, que a una lógica de construcción en la cual piensen en el texto que tienen que producir y en el tipo de lectura que éste demanda:

\footnotetext{
Estudiante 1

Normalmente cuando son trabajos finales, tengo la costumbre de poner un calendario de materias más o menos estableciéndome fechas, "en esta semana hago lectura", "en esta otra hago otras", me pongo fechas, dependiendo de las demás tareas y todo. Si no se cumplen, pues se me van juntando... si en la semana que tenía que hacer dos hice solo una, ya en la otra tengo tres.

...primero leía todo y luego hacía mis reportes de lectura y de mis reportes iba sacando la información, ya luego escribía lo que ponía y si no me gustaba lo replanteaba todo.
}

\section{Estudiante 2}

.... en cierto tiempo voy a lograr esto o voy hacer esto, con base en lo que se van necesitando las cosas se van haciendo pero no tengo estructurado como decir "en tanto tiempo se va a lograr esto, ya se logró".

En contraste, Valery (2000) afirma que la realización de operaciones asociadas a la planificación de escritura está regida por un motivo que se encuentra subordinado a una tarea y proyecto determinado mediante el cual el lector-escritor controla y estructura sus procesos de pensamiento a partir del contenido de lo que constituirá su texto.

\section{La influencia de las prácticas escolares en la lectura para tareas académicas vinculadas a la investigación. Reflexiones finales}

En la explicación de la práctica lectora de las estudiantes y de los estudiantes universitarios con fines de escritura de un texto académico como el mencionado en este 
trabajo se distinguen varias cuestiones. En primer lugar, la necesidad para el estudiantado de hacer un uso de los textos más allá de la identificación de la pertinencia temática y su reducción, esto es, la exigencia de que al leer no solamente localicen y extraigan información adecuada de acuerdo con un tema de estudio, lo cual en el modelo de análisis de tarea diseñado en este trabajo correspondería al primer nivel, la síntesis, sino que piensen en criterios de integración de esa información, dado que tareas de elaboración de un texto académico como un estado de la cuestión, además de síntesis y comprensión de los conceptos y afirmaciones requieren una mirada más profunda sobre los textos, en la cual se pueda detectar cómo las ideas y datos presentados por determinado autor se relacionan con otras, es decir, los niveles siguientes del modelo.

Acerca de lo anterior, se encontró que una influencia importante para mostrar un desempeño que evidencie estos otros niveles es la exposición del estudiantado a prácticas de uso de textos en las cuales se puede o no priorizar en aspectos como la comprensión de perspectivas y su puesta en relación, lo cual depende fundamentalmente, como sugieren en particular los datos de las entrevistas, del tipo de actividades que de manera predominante solicita el profesorado.

Habitualmente, en el contexto de donde se obtuvieron los datos de este trabajo, el estudiantado es informado al inicio de un período escolar acerca de aspectos como el programa de estudio, los autores a leer y las formas de trabajo y evaluación. Aquí se incluye la solicitud de ciertos productos escritos a entregar, la mayoría de los cuales, según indica el estudiantado, no exige una reelaboración personal del contenido de los textos. Sin embargo, por lo general no se implican en dicho programa acciones de lectura que les permitan construir textos académicos como un estado de la cuestión, posiblemente porque se da por supuesto que a este nivel los estudiantes ya saben determinar las condiciones de este tipo de textos.

Lo encontrado en los datos corrobora que las estudiantes y los estudiantes no siempre consiguen niveles de lectura que les permitan utilizar la información para tareas complejas, como la producción de un texto académico, en parte porque realizan su práctica lectora motivados por determinados fines y demandas producto de su actuación en un contexto escolar. De allí que la actuación del profesorado en la definición de esos fines sea uno de los factores importantes en la orientación de prácticas lectoras como las siguientes:

a. Leer para familiarizarse con las ideas de otros autores, esto es, como inducción a su pensamiento. 
b. Leer para hablar en clase, también denominada participación, misma que se da a través de intervenciones en lenguaje oral.

c. Leer para generar interlocución con el profesor, quien espera dialogar con los estudiantes sobre una lectura previa hecha por ambos.

Conviene señalar que en la licenciatura a la que pertenecen las estudiantes y los estudiantes que formaron parte de esta investigación ofrece una materia llamada "Expresión oral y escrita", en la cual se supone podrían adquirir herramientas para la construcción de textos. Sin embargo, como evidencian algunos señalamientos de la experiencia del estudiantado, tanto esta materia como las otras están sujetas a una lógica en la cual la información se debe reportar en un "trabajo final" que no siempre implica utilizarla junto a la reflexión personal.

Este tipo de cuestiones confirma parte de la experiencia de quienes realizamos este estudio, en cuanto a que una cultura que prevalece en las aulas universitarias donde realizamos labores formativas, una gran parte del profesorado solo señala las dificultades 0 carencias del estudiantado al leer o escribir, pero no implementa acciones para corregir o intervenir en ello. De este modo, cabe la posibilidad de que en algunos contextos de enseñanza universitaria no se requiera de una lectura o una escritura como la que se ha tipificado como ideal en este trabajo para producir un texto académico como un estado de la cuestión. En contraste, parece que lo importante es la entrega de trabajos y no la posibilidad de adquirir en el transcurso de los cursos experiencias que cuestionen y reorienten la práctica lectora y escritora.

Una de las insuficiencias de las prácticas del profesorado en ese sentido es que la revisión crítica que hacen de los textos producidos por las y los estudiantes, en muchos casos, se limita a cuestiones de ortografía y gramática, en lugar de mostrar el desarrollo de la lectura y la escritura como medio para construcción de conocimiento.

En suma, el análisis de algunos textos del estudiantado y la exploración de parte de su experiencia de formación en un área disciplinar, y sobre todo, en un contexto escolar, conduce a la necesidad de ver la práctica lectora como un ir y venir entre fines como la reproducción, la comprensión, la discusión, y en el mejor de los casos, la redacción de productos integradores del pensamiento. 


\section{Referencias}

Barton, David. (1994). The Social Impact of Literacy [El impacto social de la litarcidad]. En Ludo Verhoeven (Ed.), Functional Literacy. Theoretical issues and educational implications, [Literacidad funcional. Cuestiones teóricas y sus implicaciones educativas] (Vol. 1, pp. 185-198). Amsterdam y Philadelphia: Benjamin's Publishing Company.

Barton, David y Hamilton, Mary. (1998). Local literacies. Reading and writing in one community [Literacidades locales. Lectura y escritura en una comunidad]. London: Routledge.

Barton, David y Hamilton, Mary. (2000). Literacy practices [Prácticas de literacidad]. En David Barton, Mary Hamilton e Roz Ivaníc, Situated literacies, reading and writing in context [Literacidades situadas, lectura y escritura en contexto] (pp. 7-15). New York: Routledge.

Caldera, Reina y Bermúdez, Alexis. (2007). Alfabetización académica: Comprensión y producción de textos. Educere, 11(37), 247-255. Recuperado de http://www.saber.ula.ve/bitstream/123456789/20149/2/articulo9.pdf

Carlino, Paula. (2003a). Alfabetización académica: Un cambio necesario, algunas alternativas posibles. Revista Venezolana de Educación, 6(20), 409-420. Recuperado de http://www.saber.ula.ve/handle/123456789/19736

Carlino, Paula. (2003b). Leer textos científicos y académicos en la educación superior: Obstáculos y bienvenidas a una cultura nueva. Uni-pluri/versidad, 3(2). Recuperado de http://aprendeenlinea.udea.edu.co/revistas/index.php/unip/issue/current

Carlino, Paula. (2004). El proceso de escritura académica: Cuatro dificultades de la enseñanza universitaria. Educere, 8(26), 321-327. Recuperado de http://23118.psi.uba.ar/academica/cursos actualizacion/recursos/carlino cuatro.pdf

Carlino, Paula. (2007). ¿Qué nos dicen las investigaciones internacionales sobre la escritura en la universidad? Psicopedagogía, (4), 21-40. Recuperado de http://www.fvet.uba.ar/postgrado/especialidad/blb/Carlino Que nos dicen las investig aciones internacionales Cuad Pedag 07.pdf

Cartolari, Manuela y Carlino, Paula. (2011). Leer y tomar apuntes para aprender en la formación docente: un estudio exploratorio. Magis, Revista Internacional de Investigación en Educación, 4(7), 67-86. Recuperado de http://www.redalyc.org/pdf/2810/281021741004.pdf

Cook-Gumperz, Jenny. (1988). Alfabetización y escolarización: ¿una ecuación inmutable? En Jenny Cook-Gumperz (Ed.), La construcción social de la alfabetización (pp. 31-59). Cambridge: University Press.

Castelló, Montserrat. (2002). De la investigación sobre el proceso de composición en la enseñanza de la escritura. Revista Signos, 35(51-52), 149-162. Recuperado http://www.scielo.cl/scielo.php?script=sci arttext\&pid=S071809342002005100011\&lng=es\&nrm=iso\&t|ng=es 
Diehl, William y Mikulecky, Larry. (1988). The Nature of Reading at Work [La naturaleza de la lectura en el trabajo]. En Eugene Kingten, Barry Kroll y Mike Rose (Eds.), Perspectives on literacy [Perspectivas sobre literacidad] (pp. 190-204). USA: Sauthern Illinois University.

Dueñas, José y Jiménez, José. (Julio-Septiembre, 2006). Algunas aportaciones del análisis de tarea al estudio del desarrollo cognitivo. El caso del aprendizaje de la matemática en el bachillerato. Revista de Educación y Desarrollo, 5, 47-54. Recuperado de http://www.cucs.udg.mx/revistas/edu desarrollo/anteriores/5/005 Duenas.pdf

Escalante, Dilia y Caldera, Reina. (Enero-Diciembre, 2006). Todo docente es un maestro de lectura. El paradigma del lector estratégico. Acción Pedagógica, 15(1), 112-119. Recuperado de http://www.saber.ula.ve/handle/123456789/17264

González, Yolanda. (2011). Configuraciones de las prácticas lectoras en contextos sociales: La lectura situada en la escuela y el trabajo. Perfiles educativos, 33(133), 30-50.

Hamilton, Mary. (2000). Expanding the new literacy studies. Using photographs to explore literacy as a social practice [Ampliando los nuevos studios de literacidad. Uso de fotografías para explorar la literacidad como práctica social]. In David Barton, Mary Hamilton and Rozz Ivaníc (Eds.), Situated literacies, reading and writing in context [Literacidades situadas, lectura y escritura en context] (pp. 16-34). New York: Routledge.

Kalman, Judith. (Mayo-Julio, 2004). El estudio de la comunidad como espacio para leer y escribir. Revista Brasileira de Educação, (26), 5-28.

Luke, Carmen. (2005). Cyber-scholling and technological change. Multiliteracies for new times [Ciber-escolaridad y cambio tecnológico. Multiliteracidades para los nuevos tiempos.]. In Bill Cope y Mary Kalantzis. Multiliteracies (Eds.), Literacy learning and the design of social futures [Multiliteracidaes. Aprendizaje de la literacidad y diseño del futuro social] (pp. 69-91). New New York: Routledge.

Muñoz, Juan y Maldonado, Teresita. (2013). Dificultades para la construcción de la literacidad en la educación médica. Actualidades Investigativas en Educación, 13(2). Recuperado de http://www.scielo.sa.cr/pdf/aie/v13n2/a04v13n2.pdf

Peredo, Alicia, Leal, Fernando, Greybeck, Bárbara, Zarabozo, Daniel y González, Alicia. (2007). Lectura informativa. Entrenamiento escolar y metacognición. Guadalajara: Editorial Universitaria.

Rockwell, Elsie. (1988). Los usos escolares de la lengua escrita. En Emilia Ferreiro y Margarita Gómez (comps.), Nuevas perspectivas sobre los procesos de lectura (pp.296-320). México: Siglo Veintiuno Editores.

Rodrigo, María. (1982). Las posibilidades del análisis de tareas como técnica para el estudio de los procesos mentales. Infancia y Aprendizaje, (19-20), 159-175. Recuperado de http://dialnet.unirioja.es/servlet/articulo?codigo=668595 
Salgado, Francisco. (2007). Guía para la redacción del artículo de revisión sobre el estado de la cuestión. Recuperado de http://es.scribd.com/doc/77114653/METODO-DEREDACCION

Solé, Isabel y Castells, Nuria. (2004). Aprender mediante la lectura y la escritura.¿ Existen diferencias en función del dominio disciplinar? Lectura y Vida, 25(4), 6-17. Recuperado de http://www.lecturayvida.fahce.unlp.edu.ar/numeros/a25n4/25 04 Sole.pdf

Sticht, Thomas. (1978). The Development of Literacy [El desarrollo de la literacidad]. En Curriculum Inquiry, 8(4), 341-351.

Street, Bryan. (2004). Los nuevos estudios de literacidad. En Virginia Zavala, Mercedes NiñoMurcia y Patricia Arnés (Eds.), Escritura y sociedad. Nuevas perspectivas teóricas etnográficas (pp. 81-107). Lima: Red para el Desarrollo de las Ciencias Sociales en el Perú.

Szwed, John. (1988). The Ethnography of Literacy [La etnografía de la literacidad]. En Eugene Kingten, Barry Croll y Mike Rose (Eds.), Perspectivas on literacy [Perspectivas sobre literacidad] (pp. 303-311).USA: Sauthern Illinois University.

Valery, Olga. (2000). Reflexiones sobre la escritura a partir de Vygostky. Educere, 3(9), 3843. Recuperado de http://www.redalyc.org/articulo.oa?id=35630908

Verhoeven, Ludo. (1994). Modeling and Promoting Functional Literacy [Modelado y promoción de la literacidad]. En Ludo Verhoeven (Ed.), Functional Literacy. Theoretical issues and educational implications, [Literacidad funcional. Cuestiones teóricas e implicaciones educativas] (Vol. 1, pp. 3-34). Amsterdam y Philadelphia: John Benjamin's Publishing Company.

Zavala, Virginia. (2011). La escritura académica y la agencia de los sujetos. Cuadernos Comillas, (1), 52-66. Recuperado de http://www.cuadernoscomillas.es/pdf/5 zavala.pdf 